\title{
Pre- and Post-operative Pain Catastrophizing and Resilience of Patients With Total Knee Arthroplasty: A Cohort Study
}

\section{Vesta Nwankwo ( $\square$ vcn5@duke.edu )}

Duke University School of Medicine https://orcid.org/0000-0002-0380-4616

William A. Jiranek

Duke University School of Medicine

Cynthia L. Green

Duke University School of Medicine

Kelli D. Allen

Duke University School of Medicine

Steven Z. George

Duke University School of Medicine

Research article

Keywords: arthroplasty, knee, resilience, pain, catastrophizing, function, physical, mental, health

Posted Date: August 21st, 2020

DOI: https://doi.org/10.21203/rs.3.rs-54613/v1

License: (c) (1) This work is licensed under a Creative Commons Attribution 4.0 International License. Read Full License 


\section{Abstract}

\section{Background}

Patients undergoing total knee arthroplasty (TKA) have psychological comorbidities that influence recovery and functional outcomes. Resilience is a psychological construct defined as an individual's ability to adapt to adversity. Pain catastrophizing encompasses rumination, magnification, and helplessness that patients feel toward their pain. Pain catastrophizing is associated with poor function following surgery. Studies assessing the influence of resilience on postoperative outcomes have shown inconsistent findings and have not accounted for the influence of pain catastrophizing. We sought to identify (1) whether baseline pain catastrophizing and resilience are predictive of postoperative knee function, general physical health, and general mental health three months after TKA and (2) whether baseline pain catastrophizing and resilience are associated with preoperative knee function, general physical health, and general mental health.

\section{Methods}

Patients undergoing TKA between January 2019 and November 2019 were included in this longitudinal cohort study. Demographics and questionnaires [Brief Resilience Scale (BRS), Pain Catastrophizing Scale (PCS), Knee injury and Osteoarthritis Outcome Score, Junior (KOOS, JR.) and Patient-Reported Outcomes Measurement Information System Physical and Mental Health (PROMIS PH and MH, respectively)] were collected preoperatively and 3 months postoperatively. Multivariable regression was used to test associations of preoperative BRS and PCS with postoperative outcomes.

\section{Results}

The study cohort included 117 patients with a median age of 67.0 years (Q1-Q3: 59.0-72.0). Fifty-three percent of patients were women and $70.1 \%$ were white. In multivariable linear regression analyses, baseline pain catastrophizing did not demonstrate a significant association with postoperative 3-month knee function, $(\beta=-0.08, p=0.543)$, general physical health $(\beta=-0.11, p=0.313)$, or general mental health $(\beta=0.01, p=0.919)$. Baseline resilience positively associated with postoperative knee function ( $\beta$ $=0.24, p=0.019)$ and general physical health $(\beta=0.24, p=0.013)$.

\section{Conclusions}

Our prospective analysis suggests that resilience predicts postoperative knee function and general physical health in patients undergoing TKA. Given the inconsistent findings in this area of research, future studies should continue to delineate preoperative constructs that impact patients following surgery. Exploring interventions targeting the preoperative mental health of patients undergoing TKA may improve outcomes in this cohort.

\section{Background}


Total knee arthroplasty (TKA) is a procedure that is currently performed over 600,000 times annually in the United States [. Common indications for TKA include pain, disability, impact on daily function, and arthritic deformity of the knee such as osteoarthritis, rheumatoid arthritis, and other forms of arthritic deformity [. Osteoarthritis patients account for approximately 95\% of TKA cases each year [2]]. Typically, before surgery is considered, physicians will initiate a trial period of conservative therapies [. These can vary based on the type of arthritic insult, but can include weight loss, aerobic and anaerobic exercise, nonsteroidal anti-inflammatory drugs, and a variety of other treatments. Despite pain reduction and mobility improvements from TKA, research has indicated that patients undergoing TKA with higher levels of preoperative depression experience greater pain severity [, poorer function [5], higher levels of knee disability [5], higher dissatisfaction [], and lower self-rated health [ following surgery. Patients screening negative for depression experience higher levels of perceived function post-surgery [. Similarly, patients with higher levels of anxiety experience poorer function [5], more pain limitations [5], and higher levels of knee disability [5], while lower trait anxiety is associated with higher levels of perceived function after surgery [8].

Similar to depression and anxiety, pain catastrophizing is a negative psychological construct that has received attention in orthopedics and other fields. Pain catastrophizing captures patients' pain-related thoughts of rumination, magnification, and feelings of helplessness [. In chronic pain studies, pain catastrophizing has been cited as a vulnerability factor in the pathway to physical functioning whereas resilience mechanisms are thought to represent positive coping responses []. Further, higher resilience may predict reductions in pain catastrophizing in chronic pain patients over time [. Pain catastrophizing has been shown to negatively correlate with functional outcomes in patients with TKA [9], and a systematic review conducted in patients with TKA demonstrated a relationship between pain catastrophizing and increased chronic pain [. Higher levels of pain catastrophizing have also been linked to poor function [, more postoperative pain [, and more nighttime pain [12].

Unlike pain catastrophizing, resilience is a positive psychological construct that has recently gained more attention in orthopedics. This construct typically encompasses positive environmental and emotional characteristics that allow a person to endure adversity [. Optimism, independence, and protective family and community networks are also used to define this construct []. Psychological resilience is inversely correlated with depression and facilitates adaptation to distressing events, such as psychological and physical trauma [, ]. Only a few studies in the total joint replacement literature have attempted to define the relationship between resilience and post-surgical outcomes [, , ]. Findings to date on the relationship between resilience and postsurgical function have been inconclusive.

To date, no studies we are aware of have assessed the association between resilience and outcomes while accounting for pain catastrophizing in a cohort of patients with TKA. The primary aim of this study was to investigate whether baseline pain catastrophizing and resilience are predictive of postoperative knee function and general physical and mental health three months after TKA. The secondary aim of this study was to determine whether baseline pain catastrophizing and resilience are associated with preoperative knee function as well as general physical and mental health. 


\section{Methods}

\section{Study Design and Setting}

In this prospective cohort study, patients were recruited from an outpatient orthopedic clinic of a large academic medical center from January 2019 to November 2019. Approval from the Institutional Review Board (IRB) was obtained prior to initiation of this study. Patient information was collected and stored within REDCap, a secure, web-based application platform [[i]].

\section{Patients, Enrollment, and Study Size}

Prior to a clinic appointment, the patient's electronic medical record was pre-screened for study eligibility. Following consent for surgery by one of four joint replacement surgeons in the outpatient orthopedic clinic, patients were informed of the opportunity to participate in a study of outcomes for patients undergoing TKA. If interested and study eligible, the study was explained, and the patient consented prior to leaving the clinic. Patients were eligible if they were able to read and write in English, able to provide written informed consent, 35 to 85 years old, and approved to undergo unilateral TKA by an orthopedic surgeon. We chose not to include patients younger than 35 years in order to select against patients with knee pathology related to congenital, traumatic, and developmental origins [[ii]]. The maximum age of 85 years was chosen to minimize loss to follow-up based on the clinic's experience with electronic data collection where patients were required to participate from home after surgery. Patients were excluded based on the following criteria: medically unstable presentation at time of consent (indicating a picture of shock or sepsis), TKA scheduled because of a fracture, malignancy or an infection, bilateral TKA, cognitive and/or neurological disorders that could interfere strongly with questionnaires and surveys. Patients were further excluded if their baseline measures were incomplete.

\section{Data, Sources, and Procedures for Collection}

\section{Data Collection}

Baseline assessments were collected at least 7 days prior to the patient's surgery date. Following study enrollment, patients completed a demographics survey capturing age, sex, race, ethnicity, marital status, employment status, years of education, and insurance type. The patient's current overall pain intensity was assessed using a Pain Numeric Rating Scale (NRS). The Pain NRS is a single item response value on a scale of 0-10 with higher scores indicating increased intensity [[iii]].

At baseline, patients were asked to complete four questionnaires. These questionnaires were repeated at 3-months following surgery. At the postoperative visit, a study investigator would systematically attempt to meet patients in person at their scheduled clinic visit with the operating provider (if scheduled). If no appointment was scheduled, study investigators would utilize email, then a phone call, which was followed by a mailed survey packet if no contact was made. All follow-up data were obtained no more than 1 week before or after each scheduled follow-up time point. 
Comorbidities have been shown to influence functional outcomes of following TKA [11]. To account for these factors, baseline clinical information was collected via retrospective chart review of the electronic medical record. These data included a documented history or clinical diagnosis of depression, anxiety, and back pain (with specific category for low back pain). We also evaluated for the presence of diabetes by identifying patients with a history or clinical diagnosis of diabetes, or evidence of any of the following: blood sugar $>7 \mathrm{mmol} / \mathrm{L}$ or $>126 \mathrm{mg} / \mathrm{dL}$ on two or more fasting plasma glucose tests; blood sugar $>200$ $\mathrm{mg} / \mathrm{dL}$ on two or more oral glucose tolerance tests; blood sugar $>200 \mathrm{mg} / \mathrm{dL}$ on random plasma glucose test in the presence of increased urination, increased thirst, or unexplained weight loss; hemoglobin A1c $\geq 6.5 \%$; chronic treatment with anti-diabetic medications, including insulin. Classification for a history or clinical diagnosis of diabetes for this study did not include gestational diabetes, glycemic disorders (e.g., hypoglycemia), or pre-diabetes.

Patients were evaluated for hypertension by determining if they had a history or clinical diagnosis of high blood pressure; or evidence of any of the following: hypertension, whether treated or untreated; blood pressure $>140 \mathrm{~mm} \mathrm{Hg}$ systolic and/or $>90 \mathrm{~mm} \mathrm{Hg}$ diastolic for patients without diabetes or chronic kidney disease; blood pressure $>130 \mathrm{~mm} \mathrm{Hg}$ systolic and/or $80 \mathrm{~mm} \mathrm{Hg}$ diastolic on at least two occasions for patients with diabetes or chronic kidney disease; currently prescribed medication for treatment of hypertension (e.g., Angiotensin-converting enzyme inhibitor, angiotensin receptor blocker, beta blocker and diuretic). Finally, patients were considered to have a history of cardiovascular disease if there was documentation of a history or clinical diagnosis of coronary artery disease, myocardial infarction, stroke, arrhythmia, valvular disease, and heart failure [[iv]]. Body mass index (BMI), the American Society of Anesthesiologists (ASA) classification score ( $1=$ a normal healthy patient, $2=a$ patient with mild systemic disease, 3 = a patient with severe systemic disease, $4=$ a patient with severe systemic disease that is a constant threat to life, 5 = a moribund patient who is not expected to survive without the operation and $6=$ a declared brain-dead patient whose organs are being removed for donor purposes) [[v]], surgery type (primary vs. revision), previous TKA on the contralateral side, diagnostic criteria (knee arthritis etiology), smoking status, and pack years were also collected in the retrospective chart review.

\section{Independent Variables}

Patient resilience scores were calculated using the Brief Resilience Scale (BRS) [16]. This 6-item scale was designed to succinctly assess a patient's perception of their ability to "bounce back" in the setting of negative life events. This scale represents the concept of resilience most directly, whereas other scales are more reflective of the personality traits and strategies that patients utilize in order to increase their resilience. Items on the BRS are scored on a 5-point Likert Scale. The total BRS score (range 1 to 5) is an average of all of the items (after reverse coding 3 items) with higher scores indicating more resilient individuals.

Pain catastrophizing scores were calculated using the Pain Catastrophizing Scale (PCS) [9, [vi]]. This instrument incorporates common thoughts and reactions seen in pain catastrophizers: rumination ("I 
can't stop thinking about how much it hurts"), magnification ("I worry that something serious may happen"), and helplessness ("There is nothing I can do to reduce the intensity of the pain"). This scale is useful because it analyzes recent pain-related thoughts. The PCS score is obtained by summing the values for all 13 items within the measure. Scores range from 0-52 and higher scores indicate increased pain catastrophizing.

\section{Outcomes}

The following standardized instruments were utilized to collect patient-reported information regarding health:

Knee injury and Osteoarthritis Outcome ScoreJoint Replacement (KOOS, JR.) [[vii]]. The KOOS, JR. is a Likert-style questionnaire designed to evaluate patient's stiffness, pain ("twisting/pivoting", "straightening", "going up or down the stairs", and "standing"), and functional ability ("rising from sitting" and "bending to the floor"). Patients indicate their level of stiffness and pain/difficulty performing these tasks based on the following options: "none", "mild" "moderate", "severe", or "extreme". The KOOS Jr. questions capture patient opinions up to one week prior to survey administration. Scores are transformed to a scale ranging from $0-100$, with higher scores representing better knee function. The test has been validated against legacy measures, the Western Ontario and McMaster Universities Osteoarthritis Index (WOMAC) and full-length KOOS, which take considerably longer to administer [30]. This measure is also recommended for use with patients undergoing TKA in the perioperative period by the Centers for Medicare and Medicaid under the Comprehensive Care for Joint Replacement Model [[viii]].

Patient-Reported Outcomes Measurement Information System (PROMIS) Global Health Instrument [[ix]]. This instrument utilizes 10 items to calculate two raw scores for physical and mental health and raw response scores for the patient's perception of overall health and social health. A physical health raw score (PROMIS PH) is calculated from items that ask patients to "rate" their physical health, fatigue, and pain while also considering the patient's ability to carry out every day physical activities. Similarly, a mental health raw score (PROMIS MH) can be derived from items based on the patient's general quality of life, mood and ability to think, social satisfaction, and susceptibility to emotional problems [[x]]. T-score tables are used for comparison of the physical and mental health raw scores to the general population [[xi]]. After conversion, 50 is the mean, and converted t-scores that are 10 points below or above this number are understood to be 1 standard deviation away from the mean [33]. This allows for comparison of the mental and physical health scores to the general population with higher scores are indicating better health. The social and overall health raw response scores provide insight into the patient's perception at present, but these two items are not incorporated into composite scores [33].For these two items, responses are recorded on a 5 -point Likert Scale ranging from $1=$ poor to $5=$ excellent.

\section{Statistical Analysis}

Continuous variables are presented using the mean and standard deviation (SD) or median with $25^{\text {th }}$ and $75^{\text {th }}$ percentiles dependent on data distribution. Normality of continuous data was assessed using the 
Shapiro-Wilk test. Categorical variables are described using counts and percentages of non-missing data. Certain categorical variables were simplified into common subgroups to reduce categories and increase power.

Correlations among continuous preoperative variables were determined by calculating Pearson correlation coefficients to determine the linear relationship. Multivariable linear regression models were constructed for each 3-month postoperative primary outcome, including KOOS and PROMIS (GH and $\mathrm{MH})$. The goal was to describe the independent association between each of resilience and pain catastrophizing and the outcome variable of interest. Based on our predetermined data collection procedures we anticipated the number of missing patients would be low. Therefore, we constructed three multivariable models for knee function, general physical health, and general mental health using only complete cases. Preoperative covariates were chosen for each of three models using univariable linear regression analyses to determine the relationship of each preoperative variable (described in Table 1) to each outcome. Each covariate with a significance level of $p<0.15$ was considered and ultimately included in the multivariable models. Therefore, covariates for each model vary. This cutoff was selected to increase chances of including predictors in each final model that most appropriately explain each outcome in this cohort. Final models were assessed for multicollinearity using the variance inflation factor with a cutoff of 3 . Assumptions for multiple regression were met for each outcome (i.e., normality of the residuals, homoscedasticity, and linearity). Model results are presented as the regression slope estimate with $95 \%$ confidence interval $(\mathrm{Cl})$.

Analyses were conducted using RStudio [[xii]]. A p $<0.05$ was considered statistically significant unless otherwise indicated. Sample size was calculated assuming at least $80 \%$ power and an alpha level of 0.05 . With the inclusion of 9 predictors in a multivariable linear regression model, it was determined that 100 patients would be needed to determine an effect size of 0.080 . Additional patients were recruited assuming that some patients would be lost to follow-up.

\section{Results}

A total of 404 patients with upcoming clinic visits for surgery evaluation were screened in clinic based on chart review of eligibility criteria. Of those eligible patients, 276 candidates were excluded from inclusion or declined to participate based on rationale provided in Fig. 1. Of eligible subjects, 128 consented to participate; however, following consent, 11 patients were administratively withdrawn because of the change in their eligibility due to surgery (Fig. 1) leaving 117 patients in the final study cohort. The median number of days before surgery was 15 days (Q1-Q3:12.0-22.0). At 3-months postoperative, the overall (clinic + phone/email/mail methods) response rate was $86.3 \%$. Data were collected by phone/email/mail for $76.9 \%$ of the 117 patients contacted at 3-month follow-up. 
Table 1. Population Description $(\mathrm{N}=117)$.

\section{Variable}

Age, years

$67.0(59.0,72.0)$

Female Sex

$62(53.0)$

White Race

$81(70.1)$

Hispanic or Latino Ethnicity**

$1(0.9)$

Private Insurance

$51(43.6)$

Married or Living as Married

$83(70.9)$

Employed in or out of the home

46 (39.3)

4-Year College or higher

67 (57.3)

$\mathrm{BMl}, \mathrm{kg} / \mathrm{m} 2$

$33.1(29.0,37.7)$

ASA Class

1

2

$2(1.7)$

67 (57.3)

3

48 (41.0)

4-6

$0(0)$

Primary Surgery

$106(90.6)$

Contralateral Knee TKA

37 (31.6)

Osteoarthritis Etiology

$113(96.6)$

History of Depression

$36(30.8)$

History of Anxiety

$22(18.8)$

Smoking Status

Never Smoker

74 (63.2)

Former Smoker

37 (31.6)

Current Smoker

$6(5.1)$

Pack Years ${ }^{\star \star \star}$

$16.3(6.0,23.5)$

History of Diabetes

37 (31.6) 


\begin{tabular}{|c|c|}
\hline History of Hypertension & $72(61.5)$ \\
\hline History of Cardiovascular Disease & $21(17.9)$ \\
\hline History of Back Pain & $68(58.1)$ \\
\hline History of Low Back Pain & $56(47.9)$ \\
\hline Pain Rating & $5.0(3.0,7.0)$ \\
\hline BRS & $4.0(3.5,4.3)$ \\
\hline PCS & $13(5.0,21.0)$ \\
\hline KOOS IS & $47.5(39.6,59.4)$ \\
\hline PROMIS PH & $39.8(34.9,44.9)$ \\
\hline PROMIS MH & $50.8(45.8,56.0)$ \\
\hline PROMIS Overall Health Rating & $3.0(3.0,4.0)$ \\
\hline PROMIS Social Activity Rating & $3.0(2.0,4.0)$ \\
\hline \multicolumn{2}{|c|}{$\begin{array}{l}\text { Note: Continuous variables are presented using the median }\left(25^{\text {th }}, 75^{\text {th }} \text { percentiles }\right) \text { and categorical } \\
\text { data are displayed using counts with percentages for non-missing data unless otherwise noted. }\end{array}$} \\
\hline \multicolumn{2}{|c|}{ 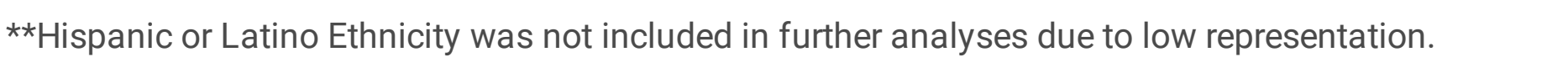 } \\
\hline \multicolumn{2}{|c|}{$\star \star \star$ Pack years not available for every patient. $\mathrm{N}=32$} \\
\hline \multicolumn{2}{|c|}{$\begin{array}{l}\text { BMI = Body mass index, ASA Class = American Society of Anesthesiologists physical status } \\
\text { classification system, BRS = Brief Resilience Score, PCS = Pain Catastrophizing Scale, Pain Rating = } \\
\text { Pain intensity on O-10 point scale, KOOS IS = KOOS interval score, PROMISPH =PROMIS Global } \\
\text { Physical Health T-score, PROMIS MH = PROMIS Global Mental Health T- score, PROMIS Overall Health } \\
\text { Rating =Raw response score from item } 1 \text { on PROMIS Global Health scale, PROMIS Social Activity } \\
\text { Rating =Raw response score from remaining item } 9 \text { on PROMIS Global Health scale. }\end{array}$} \\
\hline
\end{tabular}

- Overall, $53.0 \%$ of patients were women, and $70.1 \%$ of patients were white (Table 1 ). Of the 117 patients included in analyses, the median age was 67.0 years (Q1-Q3: 59.0-72.0). Additionally, 70.9\% of patients were married and A history of depression was seen in $30.8 \%$ of patients while $18.8 \%$ of patients were found to have a history of anxiety. A history of diabetes and low back pain were reported in $31.6 \%$ and $47.9 \%$ of patients respectively. Baseline measures of pain catastrophizing, resilience, pain, and general health are also included in Table 1.

\section{Note}

"Table 1. Population Description" will appear here in production. The table is larger than Letter page in length and has been placed at the end of the document text file.

Correlations for baseline psychological and pain variables with concurrent health and function measures are listed in Table 2. All values obtained for correlations across measures at baseline were significant. 
Pain catastrophizing was negatively correlated with knee function, while resilience positively correlated with knee function. Pain catastrophizing negatively correlated with general physical health, and resilience positively correlated with general physical health. Additionally, resilience was positively correlated with general mental health at baseline, and pain catastrophizing was negatively correlated with general mental health.

Table 2

Correlation of Baseline Patient Measures.

\begin{tabular}{|c|c|c|c|c|c|c|}
\hline & \multicolumn{2}{|c|}{ KOOS IS } & \multicolumn{2}{|c|}{ PROMIS PH } & \multicolumn{2}{|c|}{ PROMIS MH } \\
\hline & $r_{p}$ & $95 \% \mathrm{Cl}$ & $r_{p}$ & $95 \% \mathrm{Cl}$ & $r_{p}$ & $95 \% \mathrm{Cl}$ \\
\hline PCS & -0.63 & -0.51 to -0.17 & -0.55 & -0.62 to -0.32 & -0.46 & -0.52 to -0.18 \\
\hline BRS & 0.29 & 0.11 to 0.47 & 0.32 & 0.21 to 0.54 & 0.64 & 0.33 to 0.63 \\
\hline \multicolumn{7}{|c|}{$\begin{array}{l}\text { BRS }=\text { Brief Resilience Score, PCS = Pain Catastrophizing Scale, KOOS IS = KOOS interval score, } \\
P R O M I S P H=P R O M I S \text { Global Physical Health T-score, PROMIS MH }=\text { PROMIS Global Mental Health T- } \\
\text { score. }\end{array}$} \\
\hline
\end{tabular}

Simple linear regression and adjusted multiple regression models were used to determine whether pain catastrophizing predicted function and health outcomes at 3-months postoperative (Table 3a). Unadjusted analyses of baseline pain catastrophizing on knee function $(B=-0.36, p<0.001)$, general physical health $(B=-0.49, p<0.001)$ and general mental health $(B=-0.37, p<0.001)$ showed the variable was inversely predictive of these outcomes 3 months after surgery. After adjusting for resilience and other preoperative covariates, pain catastrophizing was not found to be a predictor of knee function $(\beta=-0.08$, $p=0.543)$, general physical health $(\beta=-0.11, p=0.313)$ or mental health $(\beta=0.01, p=0.919)$ at 3-months. Unadjusted analyses of baseline resilience were similarly associated with knee function, general physical and mental health (Table $3 b$ ). After adjusting for pain catastrophizing and other covariates, the significant association of resilience persisted with 3-month knee function $(\beta=0.24, p=0.019)$ and general physical health $(\beta=0.24, p=0.013)$, but not mental health at 3 months $(\beta=0.04, p=0.738)$. The full multiple regression models are displayed in Supplemental Tables $3-1$ to $3-3$ included as additional files. 
Table 3

a. Regression Models - Unadjusted and Adjusted Associations between Baseline Pain Catastrophizing and Function, Physical Health, and Mental Health at 3 months Postoperative.

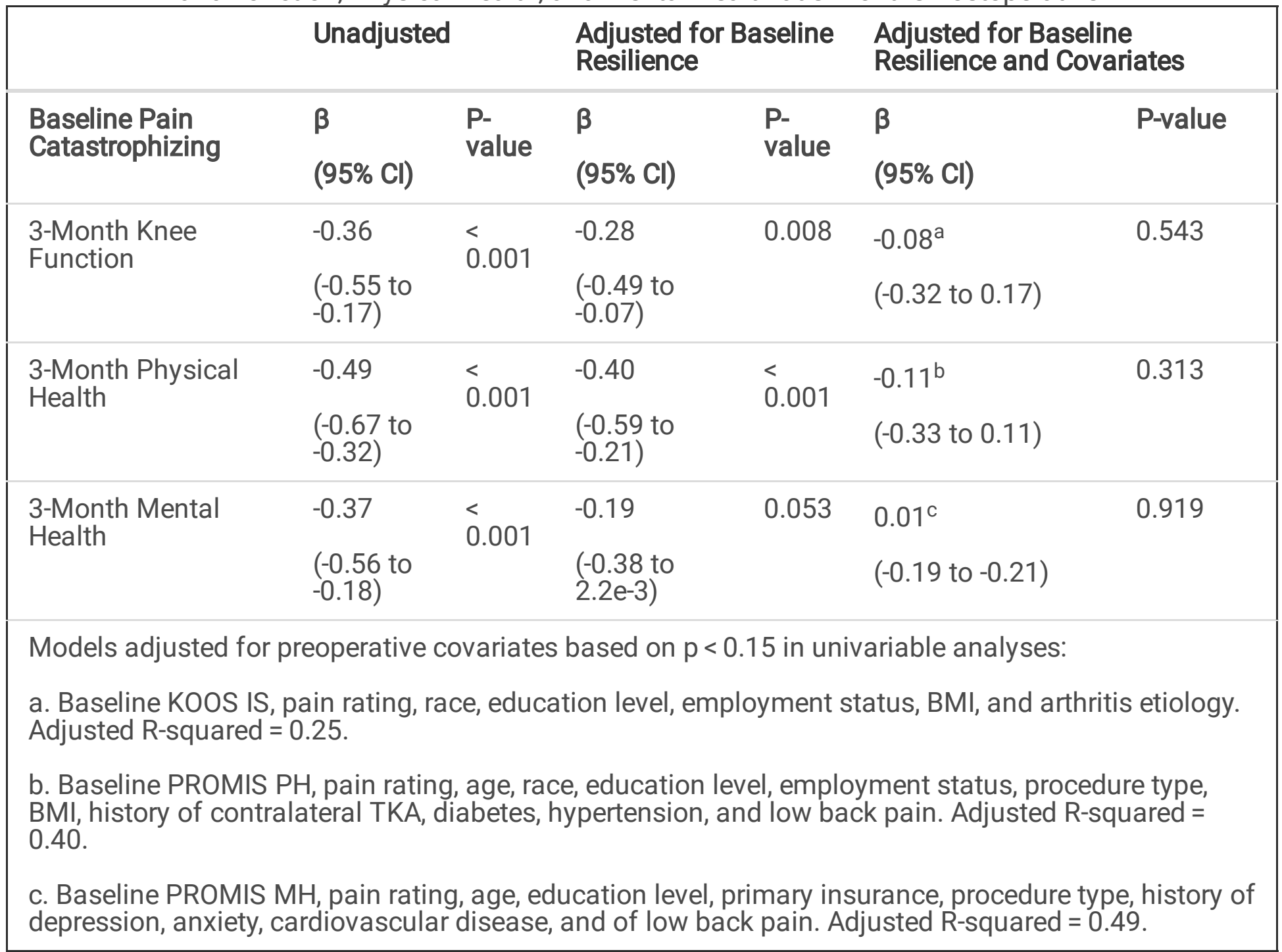


Table 3

b. Regression Models - Unadjusted and Adjusted Associations between Baseline Resilience and Function, Physical Health, and Mental Health at 3 months Postoperative.

\begin{tabular}{|c|c|c|c|c|c|c|}
\hline \multirow{3}{*}{$\begin{array}{l}\text { Baseline } \\
\text { Resilience }\end{array}$} & \multicolumn{2}{|c|}{ Unadjusted } & \multicolumn{2}{|c|}{$\begin{array}{l}\text { Adjusted for Baseline } \\
\text { Pain Catastrophizing }\end{array}$} & \multicolumn{2}{|c|}{$\begin{array}{l}\text { Adjusted for Baseline Pain } \\
\text { Catastrophizing and Covariates }\end{array}$} \\
\hline & $\beta$ & P- & $\boldsymbol{\beta}$ & P-value & $\beta$ & P-value \\
\hline & $\begin{array}{l}(95 \% \\
\mathrm{Cl})\end{array}$ & & (95\% Cl) & & $(95 \% \mathrm{Cl})$ & \\
\hline \multirow{2}{*}{$\begin{array}{l}\text { 3-Month Knee } \\
\text { Function }\end{array}$} & 0.31 & 0.002 & -0.19 & 0.074 & $0.24^{\mathrm{a}}$ & 0.019 \\
\hline & $\begin{array}{l}(0.11 \\
\text { to } \\
0.50)\end{array}$ & & $(-0.02$ to 0.40$)$ & & (0.04 to 0.44$)$ & \\
\hline \multirow{2}{*}{$\begin{array}{l}\text { 3-Month } \\
\text { Physical } \\
\text { Health }\end{array}$} & 0.40 & \multirow{2}{*}{$\begin{array}{l}< \\
0.001\end{array}$} & 0.23 & 0.018 & $0.24^{\mathrm{b}}$ & 0.013 \\
\hline & $\begin{array}{l}(0.21 \\
\text { to } \\
0.58)\end{array}$ & & (0.04 to 0.42 ) & & (0.05 to 0.42 ) & \\
\hline \multirow{2}{*}{$\begin{array}{l}\text { 3-Month } \\
\text { Mental Health }\end{array}$} & 0.51 & \multirow{2}{*}{$<0.001$} & 0.43 & $<0.001$ & $0.04^{c}$ & 0.738 \\
\hline & $\begin{array}{l}(0.33 \\
\text { to } \\
0.68)\end{array}$ & & (0.24 to 0.62$)$ & & $(-0.18$ to 0.25$)$ & \\
\hline \multirow{3}{*}{\multicolumn{7}{|c|}{$\begin{array}{l}\text { Models adjusted for preoperative covariates based on } \mathrm{p}<0.15 \text { in univariable analyses: } \\
\text { a. Baseline KOOS IS, pain rating, race, education level, employment status, BMI, and arthritis etiology. } \\
\text { Adjusted R-squared }=0.25 \text {. } \\
\text { b. Baseline PROMIS PH, pain rating, age, race, education level, employment status, procedure type, } \\
\text { BMI, history of contralateral TKA, diabetes, hypertension, and low back pain. Adjusted R-squared = } \\
0.40 \text {. }\end{array}$}} \\
\hline & & & & & & \\
\hline & & & & & & \\
\hline \multicolumn{7}{|c|}{$\begin{array}{l}\text { c. Baseline PROMIS MH, pain rating, age, education level, primary insurance, procedure type, history of } \\
\text { depression, anxiety, cardiovascular disease, and of low back pain. Adjusted R-squared }=0.49 \text {. }\end{array}$} \\
\hline
\end{tabular}

\section{Discussion}

Although no specific patient-reported outcome measure has been deemed a "gold standard" in the field of knee arthroplasty, patient experiences captured through outcome measures add valuable information to the objective and subjective clinical evaluation throughout the operative period [ii]]. In the current study, we investigated associations of pain catastrophizing and resilience with knee function and general health among patients undergoing TKA using patient reported outcome measures. Associations between these specific variables have not been widely explored. We found that greater pain catastrophizing prior to surgery was correlated with worse preoperative knee function and general health. However, at three months postoperative after adjusting for resilience and other covariates, baseline pain catastrophizing was not predictive of knee function or general health. Preoperative resilience also correlated with 
preoperative knee function and general health in a positive manner. Although no association was noted between baseline resilience and postoperative general mental health, our findings showed that baseline resilience was predictive of 3-month knee function and general physical health.

The relationship between pain catastrophizing and postoperative knee function, specifically in persons with TKA has received attention in recent years with conflicting findings. Three studies have found significant relationships between pain catastrophizing and function as measured by the WOMAC knee function subscale and postoperative function during follow-up as early as 6-weeks [9], 6-months [[ii]], and 1-year [[iii]] after adjustment. However, one study found no association after adjustment between catastrophizing and WOMAC knee function at 1-year $(n=260)$ and 5-years postoperative $(n=233)$ [[iv]]. Finally, a recent study found that patients with higher PCS scores $(>21)$ showed more improvement than patients with lower PCS scores (<11) with regard to function on the Oxford Knee Score at 12-months postoperative [[v]]. In the current study, pain catastrophizing and knee function were not significantly associated, which differs from expectations based on previous studies. There was also no significant association between pain catastrophizing and the PROMIS general health components. This is the first study we are aware of to analyze pain catastrophizing and associations with PROMIS physical health and mental health components. Previous studies following patients with TKA that utilized the Short Form Health Questionnaires (SF-12 and SF36) and EuroQol 5D demonstrated a significant relationship between pain catastrophizing and quality of life, which was not seen in our findings [[vi]]. Of note, in this study as additional variables were considered in the models, the associations between pain catastrophizing and the outcomes were attenuated in each model. We also included a different combination of variables compared to other studies, notably the resilience variable. When including covariates, the association between pain catastrophizing and outcomes is diminished, which limits the conclusions we are able to make solely based on the relationship between pain catastrophizing and resilience. Future studies can add to this interpretation through greater patient diversity. Additionally, these findings don't account for the patient experience during the acute postoperative period where patients [[vii]].

Our findings demonstrated that resilience was significantly correlated with preoperative knee function and general mental and physical health. We also found a significant association between baseline resilience and knee function and general physical health at 3-months postoperatively. Though statistically significant, clinical significance was marginal for both of these associations based on previous studies [31,[viii]]. No association was found between preoperative resilience and postoperative general mental health. Few studies have attempted to establish a relationship between resilience and postoperative outcomes. Rebagliati et. al [21] studied patients who underwent elective or traumatic hip and knee surgery. Though the study found no relationship between resilience as measured by the Resilience Scale [[ix]] and functional independence, the number of knee patients in this cohort was undefined which limits conclusions that can be drawn based on this cohort and our ability to compare findings. A second study by this research group using the same resilience scale found that the level of presurgical resilience did not relate to functional independence for patients who had undergone elective joint replacement surgery [22]. This study did identify a negative relationship between preoperative resilience and functional independence in patients who underwent joint replacement due to fracture 
$(n=80$, TKA patients $=7$, THA patients $=73$ ). These findings could be attributed to baseline characteristics of the patients assessed in these two different groups such as age or gender (specific data on both variables were not reported). In a recent study, Magaldi et. al [23] demonstrated that baseline resilience (measured by the BRS) was not related to the KOOS JR. at 3- or 12-months postoperative. However, the investigators found that baseline resilience was related to PROMIS physical health and mental health scores at 3-month and 12-month follow-up. This difference may be due to the incorporation of pain catastrophizing in the current study. This addition could be reducing the influence of the resilience variable, particularly in the PROMIS MH model.

Considering the breadth of information regarding mental health and the relationship with outcomes after TKA, it is suggested that further research is warranted in this field to further elicit other key variables [[x], [xi],[xii],[xiii]]. Determining which baseline variables significantly and consistently predict postoperative outcomes will allow clinicians to gain insights about their patients that may not have been considered broadly previously. National healthcare and orthopedic organizations have collaborated in the last decade to improve delivery of patient reported outcomes to assess quality of healthcare while also limiting patient burden in the process [[xiv]]. Acknowledging this effort and the findings from additional studies in the field, future directions should include the continued development of targeted perioperative interventions that address these constructs. Two recent studies sought to improve functional outcomes in pain catastrophizing patients undergoing TKA using pain coping skills training [[xv]] and cognitive behavioral therapy [[xvi]]. Neither study was able to demonstrate that the selected interventions were superior to usual care. However, to our knowledge, there are no studies in the total joint literature that have implemented interventions in patients with low resilience to evaluate postoperative function.

The current study is limited mostly by factors which were related to the most efficient and practical methods of collecting data from this prospective patient cohort in this single-center setting [[xvii],[xviii]]. Patients were excluded based on English proficiency. This limits the generalizability of our study to more diverse patient populations. However, standardized measures were not available in a variety of languages for each of the measures and interpreters were not available to study staff for each data collection time point, necessitating the use of the English versions. There may have also been an element of social desirability bias during visits that occurred in clinic during enrollment and initial questionnaire administration with the study investigator. This study also has strengths that should be considered. Enrollment was conducted primarily by the same individual, which contributes to the consistency of delivery of study details and answering of patient questions. To prevent loss of patients to follow-up, the team prioritized the patients' post-surgery visits in clinic to complete follow-up measures. Additionally, all patients provided complete contact information (email address, phone number, and mailing addresses) upon enrolling in the study which facilitated subsequent data collection. Patients in the study were screened consecutively to limit selection bias. Additionally, the patients of four providers within the practice were utilized in order to increase generalization of the results. Investigators also made effort to limit confounding in analyses by considering other variables that may influence associations. 


\section{Conclusions}

In conclusion, this study contributes new knowledge on pain catastrophizing and resilience, particularly their effect on subsequent outcomes of knee arthroplasty patients when analyzed together. Pain catastrophizing and resilience did show patterns of influence on outcomes. Ultimately, our findings indicated that preoperative resilience was associated with 3-month patient-reported outcomes when we consider other demographic and medical factors. There are conflicting results in this area of research, therefore the results of our study should be used to continue to delineate a model of our primary variables, as well as overall mental health within the context of total knee arthroplasty.

\section{Abbreviations}

ASA: American Society of Anesthesiologists

B: Unstandardized Beta

BMI: Body mass index

BRS: Brief Resilience Score

Cl: Confidence interval

IRB: Institutional Review Board

IQR: Interquartile range

KOOS IS: KOOS interval score

KOOS, Jr.: Knee injury and Osteoarthritis Outcome Score Joint Replacement

NRS: Pain Numeric Rating Scale

PCS: Pain Catastrophizing Scale

PROMIS: Patient-Reported Outcomes Measurement Information System

PROMIS MH: PROMIS Global Mental Health T- score

PROMIS PH: PROMIS Global Physical Health T-score

Q1: 25th Quartile

Q3: 75th quartile

$r_{p}$ : Pearson correlation coefficient 
SD: Standard deviation

SF: Short Form

TKA: Total nee arthroplasty

WOMAC: Western Ontario and McMaster Universities Osteoarthritis Index

$\beta$ : Standardized Beta

\section{Declarations}

Ethics approval and consent to participate

- Informed written consent was obtained from all participants upon their inclusion in the study. The study was approved by the DUHS IRB in Durham, North Carolina on 1/15/2019 (reference ID: 294616), in accordance with all applicable Health Insurance Portability and Accountability Act ("HIPAA") regulations.

Consent for publication

- Not applicable

Availability of data and materials

- The datasets used and/or analyzed during the current study are available from the corresponding author on reasonable request.

Competing interests

- VCN was partially supported by the National Center for Advancing Translational Sciences of the National Institutes of Health under Award Number UL1TR002553. This manuscript is solely the responsibility of the authors and does not necessarily represent the official views of the National Institutes of Health.

\section{All other authors have no competing interests.}

- WAJ is a consultant and has received royalties for hip and knee products from Depuy Synthes, A Johnson \& Johnson Company for work not related to this submitted article.

- WAJ has stock and/or stock options in Biomech Holdings LLC, a company not related to this research.

- KDA, SZG, JPG have received funding from the National Institutes of Health and Patient Centered Outcomes Research Institute for research not directly related to the submitted article. 
- JPG has received funding from the Department of Veterans Affairs for research not directly related to the submitted article.

- KDA has received funding from the Department of Defense and the Department of Veterans Affairs for research not directly related to the submitted article.

- SZG has received payments from Rehab Essentials, Inc. that is not related to the submitted article.

- CLG, SZG, KDA, JPG have no commercial associations (e.g., consultancies, stock ownership, equity interest, patent/licensing arrangements, etc.) that might pose a conflict of interest in connection with the submitted article.

\section{Funding}

- Our study was primarily supported by the institution of the first author through institutional funding. VCN was also partially supported by the National Center for Advancing Translational Sciences of the National Institutes of Health under Award Number UL1TR002553. This manuscript is solely the responsibility of the authors and does not necessarily represent the official views of the National Institutes of Health.

\section{Authors' contributions}

- All of the contributing authors were involved with drafting the proposal and revision. VCN, JPB, WAJ, and SZG were primarily involved with conception and design. VCN and JPB were primarily involved with acquisition of the data. VCN and CLG contributed to the analysis of the data. VCN primarily drafted the manuscript. All authors contributed to the interpretation of the data, revising, and approval of the submitted version. All authors assure the integrity of the manuscript.

\section{Acknowledgements}

We would like to thank faculty member, Carl Pieper, PhD, and research staff, Thomas Risoli Jr., MS, for assisting with the statistical analysis and presentation of data in this manuscript. We also thank Katherine Plampton and institution affiliates for administrative assistance.

\section{References}

1. [1] Sloan M, Premkumar A, Sheth NP. Projected Volume of Primary Total Joint Arthroplasty in the U.S., 2014 to 2030. The Journal of bone and joint surgery American volume. 2018;100:1455-1460.

2. [1]Van Manen MD, Nace J, Mont MA. Management of Primary Knee Osteoarthritis and Indications for Total Knee Arthroplasty for General Practitioners. The Journal of the American Osteopathic Association. 2012;112:709-715.

3. [1]Mahomed NN, Barrett J, Katz JN, Baron JA, Wright J, Losina E. Epidemiology of total knee replacement in the United States Medicare population. The Journal of bone and joint surgery American volume. 2005;87:1222-1228. 
4. [1] Jevsevar DS. Treatment of osteoarthritis of the knee: evidence-based guideline, 2 nd edition. The Journal of the American Academy of Orthopaedic Surgeons. 2013;21:571-576.

5. [1] Alattas SA, Smith T, Bhatti M, Wilson-Nunn D, Donell S. Greater pre-operative anxiety, pain and poorer function predict a worse outcome of a total knee arthroplasty. Knee Surg Sports Traumatol Arthrosc. 2017;25:3403-3410.

6. [1] Dhurve K, Scholes C, El-Tawil S, Shaikh A, Weng LK, Levin K, Fritsch B, Parker D, Coolican M. Multifactorial analysis of dissatisfaction after primary total knee replacement. Knee. 2017;24:856862.

7. [1] Lindberg MF, Rustoen T, Miaskowski C, Rosseland LA, Lerdal A. The relationship between pain with walking and self-rated health 12 months following total knee arthroplasty: a longitudinal study. BMC Musculoskelet Disord. 2017;18:1414-1419.

8. [1] Cooper NA, Rakel BA, Zimmerman B, Tonelli SM, Keela AH, Clark CR, Noiseux NO, Callaghan JJ, Sluka KA. Predictors of multidimensional functional outcomes after total knee arthroplasty. $J$ Orthop Res. 2017;35:2790-2798.

9. [1] Sullivan M, Tanzer, Stanish W, Fallaha M, Keefe FJ, Simmonds M, Dunbar M. Psychological determinants of problematic outcomes following Total Knee Arthroplasty. 2009;143:123-9.

10. [1]Sturgeon JA, Zautra AJ. Psychological resilience, pain catastrophizing, and positive emotions: perspectives on comprehensive modeling of individual pain adaptation. Curr Pain Headache Rep. 2013;17:317.

11. [1] Ong AD, Zautra AJ, Reid MC. Psychological resilience predicts decreases in pain catastrophizing through positive emotions. Psychol Aging. 2010;25:516-523.

12. [1] Burns LC, Ritvo SE, Ferguson MK, Clarke H, Seltzer Z, Katz J. Pain catastrophizing as a risk factor for chronic pain after total knee arthroplasty: a systematic review. Journal of Pain Research. 2015;8:21-32.

13. [1] Lungu E, Vendittoli PA, Desmeules F. Preoperative Determinants of Patient-reported Pain and Physical Function Levels Following Total Knee Arthroplasty: A Systematic Review. Open Orthop J. 2016;10:213-231.

14. [1] Edwards RR, Haythornthwaite JA, Smith MT, Klick B, Katz JN. Catastrophizing and depressive symptoms as prospective predictors of outcomes following total knee replacement. Pain Res Manag. 2009;14:307-311.

15. [1]Sturgeon JA, Zautra AJ. Resilience: A New Paradigm for Adaptation to Chronic Pain. Current pain and headache reports. 2010;14:105-112.

16. [1]Smith BW, Dalen J, Wiggins K, Tooley E, Christopher P, Bernard J. The Brief Resilience Scale: Assessing the Ability to Bounce Back. International Journal of Behavioral Medicine. 2008;15:194200.

17. [1]Zautra AJ, Arewasikporn A, Davis MC. Resilience: Promoting Well-Being Through Recovery, Sustainability, and Growth. Research in Human Development. 2010;7:221-238. 
18. [1]Suffeda A, Meissner W, Rosendahl J, Guntinas-Lichius O. Influence of depression, catastrophizing, anxiety, and resilience on postoperative pain at the first day after otolaryngological surgery: A prospective single center cohort observational study. Hanaoka. K, ed. Medicine. 2016;95:e4256.

19. [1] Duggal D, Sacks-Zimmerman A, Liberta T. The Impact of Hope and Resilience on Multiple Factors in Neurosurgical Patients. Muacevic A, Adler JR, eds. Cureus. 2016;8:e849.

20. [1] Wu, HC. The protective effects of resilience and hope on quality of life of the families coping with the criminal traumatisation of one of its members. Journal of Clinical Nursing.2011;20:1906-1915.

21. [1] Rebagliati GA, Sciume L, lannello P, Mottini A, Antonietti A, Caserta VA, Gattoronchieri V, Panella L, Callegari C. Frailty and resilience in an older population. The role of resilience during rehabilitation after orthopedic surgery in geriatric patients with multiple comorbidities. Funct Neurol. 2016;31:171177.

22. [1] Sciume L, Rebagliati GAA, lannello P, Mottini A, Alessandro A, Caserta AV, Gattoronchieri V, Panella L. Rehabilitation After Urgent or Elective Orthopedic Surgery: The Role of Resilience in Elderly Patients. Rehabil Nurs. 2018;43:267-274.

23. [1] Magaldi RJ, Staff I, Stovall AE, Stohler SA, Lewis CG. Impact of Resilience on Outcomes of Total Knee Arthroplasty. The Journal of Arthroplasty. 2019;34:2620-2623.e2621.

24. [1] Harris PA, Taylor R, Thielke R, Payne J, Gonzalez N, Conde JG. Research electronic data capture (REDCap) - A metadata-driven methodology and workflow process for providing translational research informatics support. Journal of Biomedical Informatics. 2009;42:377-381.

25. [1] Schreurs BW, Hannink G. Total joint arthroplasty in younger patients: heading for trouble? The Lancet. 2017;389:1374-1375.

26. [1] Haefeli M, Elfering A. Pain assessment. Eur Spine J. 2006;15 Suppl 1(Suppl 1):S17-S24.

27. [1] What is Cardiovascular Disease? American Heart Association. Updated May 31, 2017. Accessed November 15, 2019. https://www.heart.org/en/health-topics/consumer-healthcare/what-iscardiovascular-disease.

28. [1] Hurwitz EE, Simon M, Vinta SR, Zehm CF, Shabot SM, Minhajuddin A, Abouleish AE. Adding Examples to the ASA-Physical Status Classification Improves Correct Assignment to Patients. 2017;126:614-622.

29. [1] Sullivan MJL, Bishop SR, Pivik J. The Pain Catastrophizing Scale: Development and validation. Psychol Assessment. 1995;7:524-532.

30. [1]Lyman S, Lee Y-Y, Franklin PD, Li W, Cross MB, Padgett DE. Validation of the KOOS, JR: A Shortform Knee Arthroplasty Outcomes Survey. Clin Orthop Relat Res. 2016;474:1461-1471.

31. [1] Centers for M, Medicaid Services HHS. Medicare Program; Comprehensive Care for Joint Replacement Payment Model for Acute Care Hospitals Furnishing Lower Extremity Joint Replacement Services. Final rule. Fed Regist. 2015;80:73273-73554.

32. [1] Cella D, Riley W, Stone A, Rothrock N, Reeve B, Yount S, Amtmann D, Bode R, Buysse D, Choi S, Cook K, Devellis R, DeWalt D, Fries JF, Gershon R, Hahn EA, Lai J-S, Pilkonis P, Revicki D, Rose M, Weinfurt K, Hays R, PROMIS Cooperative Group. The Patient-Reported Outcomes Measurement 
Information System (PROMIS) developed and tested its first wave of adult self-reported health outcome item banks: 2005-2008. J Clin Epidemiol. 2010;63:1179-1194.

33. [1] Global Health: A brief guide to the PROMIS@ Global Health instruments. Health Measures. March 6, 2017. Accessed January 20, 2019. http://www.healthmeasures.net/index.php? option=com_instruments\&view=measure\&id=778\&ltemid=992.

34. [1] Hays RD, Bjorner JB, Revicki DA, Spritzer KL, Cella D. Development of physical and mental health summary scores from the patient-reported outcomes measurement information system (PROMIS) global items. Qual Life Res. 2009;18:873-880.

35. [1] RStudio Team (2016). RStudio: Integrated Development for R. RStudio, Inc., Boston, MA URL http://www.rstudio.com/.

36. [1] Ramkumar PN, Harris JD, Noble PC. Patient-reported outcome measures after total knee arthroplasty: a systematic review. Bone \& joint research. 2015;4:120-127.

37. [1] Riddle DL, Wade JB, Jiranek WA, Kong X. Preoperative pain catastrophizing predicts pain outcome after knee arthroplasty. Clin Orthop Relat Res. 2010;468:798-806.

38. [1] Sullivan M, Tanzer M, Reardon G, Amirault D, Dunbar M, Stanish W. The role of presurgical expectancies in predicting pain and function one year following total knee arthroplasty. 2011;152:2287-2293.

39. [1] Wylde V, Trela-Larsen L, Whitehouse MR, Blom AW. Preoperative psychosocial risk factors for poor outcomes at 1 and 5 years after total knee replacement. Acta Orthop. 2017;88:530-536.

40. [1] Birch S, Stilling M, Mechlenburg I, Hansen TB. The association between pain catastrophizing, physical function and pain in a cohort of patients undergoing knee arthroplasty. $B M C$ Musculoskeletal Disorders. 2019;20:421.

41. [1] Yakobov E, Stanish W, Tanzer M, Dunbar M, Richardson G, Sullivan MJL. The prognostic value of pain catastrophizing in health-related quality of life judgments after total knee arthroplasty. Health Qual Life Outcomes. 2018;16:126.

42. [1] Roth ML, Tripp DA, Harrison MH, Sullivan M, Carson P. Demographic and psychosocial predictors of acute perioperative pain for total knee arthroplasty. Pain Res Manag. 2007;12:185-194.

43. [1] Khalil LS, Darrith B, Franovic S, Davis JJ, Weir RM, Banka TR. Patient-Reported Outcomes Measurement Information System (PROMIS) Global Health Short Forms Demonstrate Responsiveness in Patients Undergoing Knee Arthroplasty. The Journal of Arthroplasty.

44. [1] Wagnild GM, Young HM. Development and psychometric evaluation of the Resilience Scale. $J$ Nurs Meas. 1993;1:165-178.

45. [1] Ali A, Lindstrand A, Sundberg M, Flivik G. Preoperative Anxiety and Depression Correlate With Dissatisfaction After Total Knee Arthroplasty: A Prospective Longitudinal Cohort Study of 186 Patients, With 4-Year Follow-Up. The Journal of Arthroplasty. 2017;32:767-770.

46. [1] Khatib Y, Madan A, Naylor JM, Harris IA. Do Psychological Factors Predict Poor Outcome in Patients Undergoing TKA? A Systematic Review. Clin Orthop Relat Res. 2015;473:2630-2638. 
47. [1] Clement N. Patient factors that influence the outcome of total knee replacement: A Critical Review of the Literature. OA Orthopaedics. 2013;1:11.

48. [1] Vissers MM, Bussmann JB, Verhaar JAN, Busschbach JJV, Bierma-Zeinstra SMA, Reijman M. Psychological Factors Affecting the Outcome of Total Hip and Knee Arthroplasty: A Systematic Review. Seminars in Arthritis and Rheumatism. 2012;41:576-588.

49. [1] Patient-Reported Outcomes Summit for Total Joint Arthroplasty Report: Double Tree by Hilton Baltimore North-Pikesville (August 31, 2015). The Journal of Arthroplasty. 2015;30:1860-1862.

50. [1] Riddle DL, Keefe FJ, Ang DC, Slover J, Jensen MP, Bair MJ, Kroenke K, Perera RA, Reed SD, McKee D, Dumenci L. Pain Coping Skills Training for Patients Who Catastrophize About Pain Prior to Knee Arthroplasty: A Multisite Randomized Clinical Trial. J Bone Joint Surg Am. 2019;101:218-227.

51. [1] Birch S, Stilling M, Mechlenburg I, Hansen TB. No effect of cognitive behavioral patient education for patients with pain catastrophizing before total knee arthroplasty: a randomized controlled trial. Acta Orthop. 2020;91:98-103.

52. [1] Althubaiti A. Information bias in health research: definition, pitfalls, and adjustment methods. $J$ Multidiscip Healthc. 2016;9:211-217.

53. [1] Ranstam J. Bias in observational studies. Acta Radiologica. 2008;49:644-645.

\section{Figures}




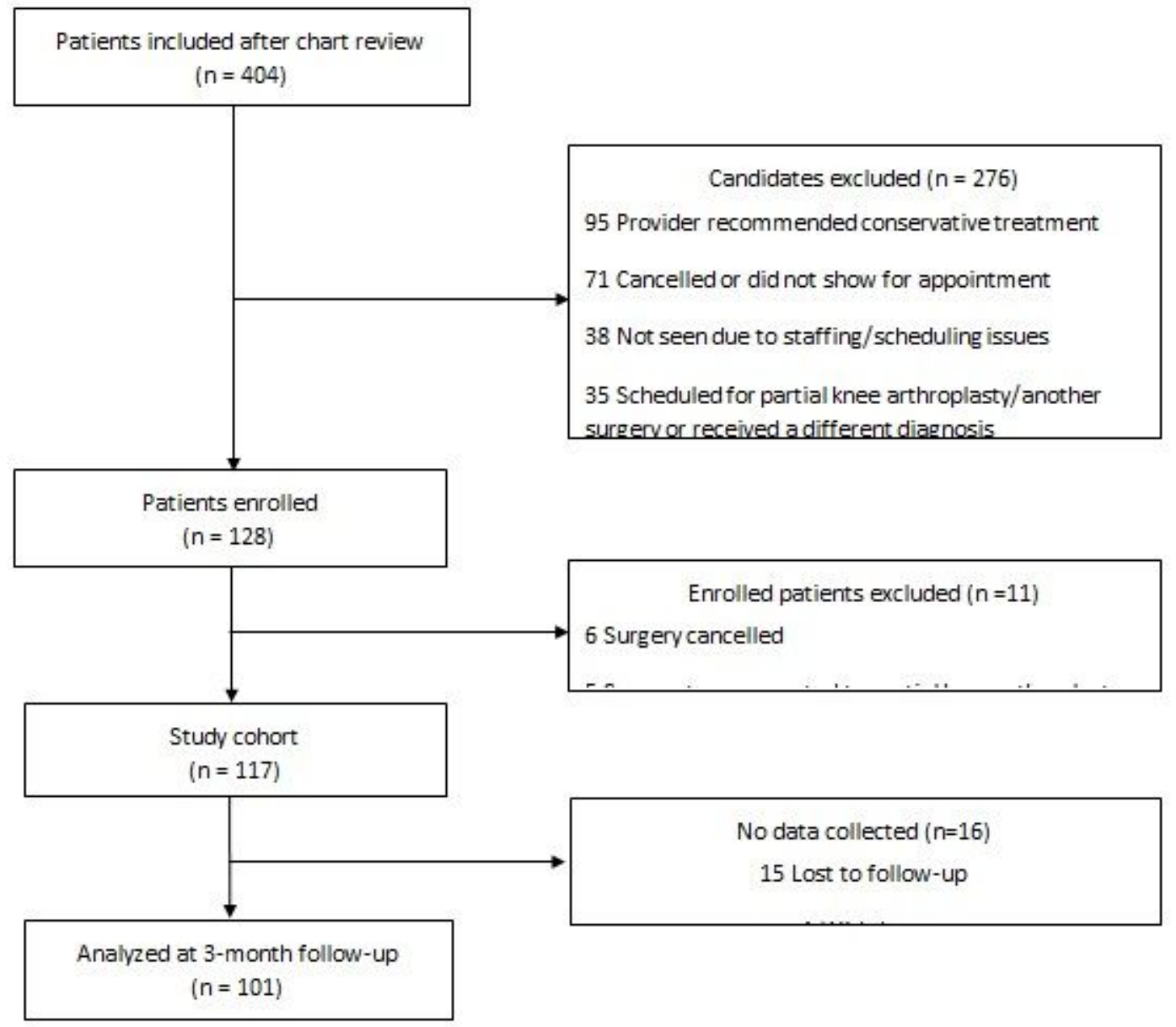

\section{Figure 1}

Flow diagram of patients in study.

\section{Supplementary Files}

This is a list of supplementary files associated with this preprint. Click to download.

- Coverletter.vcn.docx

- AdditionalFile3.Supplemenaltable33.docx

- AdditionalFile2.Supplementaltable32.docx

- AdditionalFile1.Supplementaltable31.docx 